\title{
Scattering and conductance quantization in three-dimensional metal nanocontacts
}

\author{
Brandbyge, Mads; Jacobsen, Karsten Wedel; Nørskov, Jens Kehlet
}

Published in:

Physical Review B

Link to article, DOI:

10.1103/PhysRevB.55.2637

Publication date:

1997

Document Version

Publisher's PDF, also known as Version of record

Link back to DTU Orbit

Citation (APA):

Brandbyge, M., Jacobsen, K. W., \& Nørskov, J. K. (1997). Scattering and conductance quantization in threedimensional metal nanocontacts. Physical Review B, 55(4), 2637-2650.

https://doi.org/10.1103/PhysRevB.55.2637

\section{General rights}

Copyright and moral rights for the publications made accessible in the public portal are retained by the authors and/or other copyright owners and it is a condition of accessing publications that users recognise and abide by the legal requirements associated with these rights.

- Users may download and print one copy of any publication from the public portal for the purpose of private study or research.

- You may not further distribute the material or use it for any profit-making activity or commercial gain

- You may freely distribute the URL identifying the publication in the public portal

If you believe that this document breaches copyright please contact us providing details, and we will remove access to the work immediately and investigate your claim. 


\title{
Scattering and conductance quantization in three-dimensional metal nanocontacts
}

\author{
Mads Brandbyge \\ CAMP, Department of Physics, Technical University of Denmark, DK-2800 Lyngby, Denmark \\ Karsten W. Jacobsen \\ CAMP, Department of Physics, Technical University of Denmark, DK-2800 Lyngby, Denmark, \\ and Laboratory of Atomic and Solid State Physics, Cornell University, Ithaca, New York 14853 \\ Jens K. Nørskov \\ CAMP, Department of Physics, Technical University of Denmark, DK-2800 Lyngby, Denmark
}

(Received 5 August 1996)

\begin{abstract}
The transmission through three-dimenstional nanocontacts is calculated in the presence of localized scattering centers and boundary scattering using a coupled-channel recursion method. Simple confining potentials are used to investigate how robust the observation of quantized conductance is with respect to the scattering. We find that the quantum features are quite stable: the scattering by a localized scatterer will selectively smear and downshift certain quantum steps depending on the position of the scatterer, but the remaining steps will still be at integer positions. The effect of scattering by surface corrugation depends on the length scale of the corrugation. In some cases a significant downshift of the steps without accompanying smearing is observed. In general, we find that even in the cases where scattering shifts the quantum steps the quantization of the motion perpendicular to the contacts remains intact. Non-integer steps can therefore also be a signature of quantized conductance. [S0163-1829(97)05804-9]
\end{abstract}

\section{INTRODUCTION}

The electrical and mechanical properties of nanostructures have been studied intensely over the past decade due to the possibility of new quantum properties appearing at the small scale. One class of nanostructures consists of the nanocontacts or quantum point contacts in which the transport between two leads or reservoirs are controlled at the nanoscale. These have been synthesized by lithographic techniques on semiconductor structures, e.g., GaAs- $\mathrm{Al}_{x} \mathrm{Ga}_{1-x} \mathrm{As}$, where electrons are confined to move in two dimensions. ${ }^{1,2}$ In three dimensions atomic scale metal contacts connecting two metals have been investigated. Apart from the fundamental interest in new quantum effects the understanding of these atomic sized contacts between two materials underlies the understanding of the macroscopic contact phenomena such as friction, wear, and adhesion. The experimental techniques have in the past few years progressed substantially towards measuring the electrical and mechanical properties of metallic nanoscale contacts. Two closely related experimental setups have been used in these studies. In one setup a scanning tunneling microscope (STM) is used to study contact formation/breaking between a tip and a sample metal surface. $^{3-15}$ The other technique ${ }^{16-21}$ is the so-called mechanically controllable break junction (MCB) technique, where a thin metal wire is broken by piezocontrolled bending and subsequently brought back into contact.

Both the STM and the MCB studies give information about the electrical conductance through the contacts. In some recent STM experiments ${ }^{12,13}$ the forces acting between the electrodes have also been measured along with the con- ductance. In a typical experiment the contact formation is initiated by a rapid increase in the conductance seen in both STM (Refs. 3,4,6,22,23) and MCB (Refs. 16,20) experiments. This increase can be caused by a mechanical instability ("jump to contact") ${ }^{24}$ or at higher temperatures by a cascade of diffusion processes ("diffusion to contact") ${ }^{25}$ As the contact is established the electron transport goes from the tunneling regime to the so-called ballistic regime, where the conductance is determined by the elastic quantum transmission properties of the contact. The initial rapid contact formation may be followed by a further increase in the contact area because of diffusion processes or because of continued indentation. By reversing the motion of the electrodes the contact can be pulled apart in a process where the contact gets thinner and more elongated.

The most striking feature in the measured conductance vs retraction distance is a profound step structure. This has been seen using the STM type of experiment at liquid Helium temperature for $\mathrm{Au}$ (Refs. 7,14), at room temperature for $\mathrm{Cu}$, Pt, Ni (Ref. 8), and Au (Refs. 10,14,15), and in ultrahigh vacuum at liquid nitrogen temperature for $\mathrm{Au}$ (Ref. 14). It has also been reported for $\mathrm{Au}$ in atmospheric pressure and room temperature. ${ }^{6,9,13}$ In Fig. 1 we show the conductance measured during the retraction of the STM tip. With the MCB technique step structure in the conductance has been observed for a range of systems covering the metals $\mathrm{Al}, \mathrm{Cu}$, Pt (Ref. 16) and $\mathrm{Na}$ (Ref. 18) at liquid helium temperatures, as well as the semimetallic Sb (Ref. 17). Also roomtemperature measurements ${ }^{19}$ have been performed in vacuum on $\mathrm{Cu}$ and $\mathrm{Au}$ where a step structure was observed. Recently, steps in the conductance during the breaking of a contact 


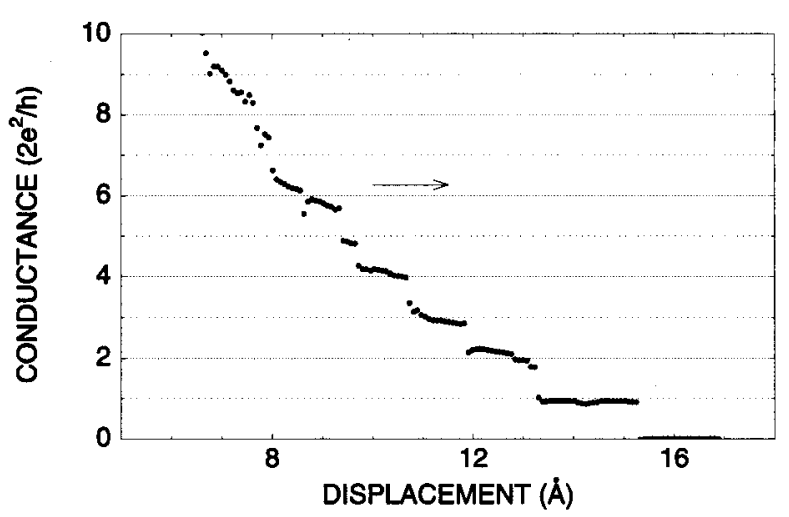

FIG. 1. The measured conductance of the contact between a STM tip and a Au surface during the tip retraction (Ref. 10).

have also been seen in a much simpler type of experiment: ${ }^{26}$ Two pieces of ordinary macroscopic wires $(\mathrm{Au}, \mathrm{Cu}, \mathrm{Pt}$, and metallic glass) are brought in loose contact with each other and by tapping the table top the contact between the wires is broken. The measured conductance between the wires shows a step structure during the last stages of contact, where only one single nanocontact is likely to be present. Now techniques derived form this method are emerging (pin-plate ${ }^{27}$, relay $\left.^{28}\right)$.

The origin of the conductance steps has been debated vividly. ${ }^{29,30}$ On the one hand it can easily be estimated that the size of the neck/wire structure in the last stages before it eventually breaks is at the very atomic scale with just a few atoms in the contact area. In this regime quantum phenomena can be expected at room temperature or even higher just as the quantum size phenomena seen in the stability of small metal clusters. ${ }^{31}$ If the contacts posses reasonably welldefined quantum modes perpendicular to the direction of transport the conductance can be expected to change in steps of the quantum unit of conductance, $G_{0}=2 e^{2} / h(1 /(12.9$ $k \Omega)$ ). This is in analogy to the experiments ${ }^{1,2}$ where a smooth constriction separating a two-dimensional (2D) electron gas is varied in diameter and the conductance through the system is quantized. Thus the origin of the steps could be a true quantum size effect.

On the other hand there are also indications that mechanical instabilities of the atomic structure of the tip play an active role during the breaking of a contact. It is clear both from computer simulations ${ }^{32-35,10}$ and from recent STM experiments with force measurements ${ }^{13}$ that the atomic neck which constitutes the contact undergoes abrupt structural changes during the breaking and the area of the cross section of the contact will therefore change in a stepwise manner. It has therefore also been suggested ${ }^{34}$ that the discontinuous change in contact area and atomic structure could be the main underlying reason for the observed steps in the conductance curves.

At the present time there is rather clear experimental evidence that for some systems the conductance just before the breaking of the contact is truly quantized. It is clear that in indentation/retraction experiments the detailed atomic structure will change from one indentation/retraction to another and the behavior of the conductance vs stretch of the contact

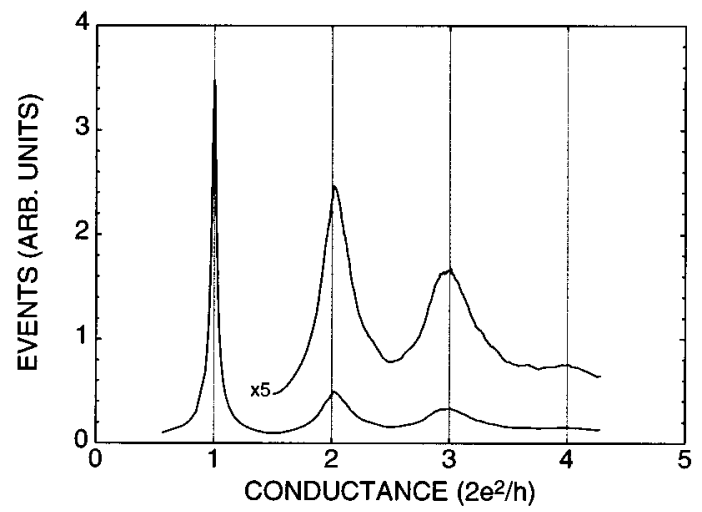

FIG. 2. A continuous conductance histogram based on 227 indentations of a STM tip on a $\mathrm{Au}(110)$ surface. Peaks at 1,2, and 3 times the quantum unit of conductance $\left(G_{0}=2 e^{2} / h\right)$ are seen. The results are taken from Ref. 10).

is therefore not reproducible. The positions of the steps will, in general, also fluctuate. However, for a range of metals the measured conductances have been seen to group around integer values times $G_{0}$. This is most clearly seen if a histogram of measured conductances during pulloff of many contacts ( $\sim 100$ or more) is constructed. Peaks at 1,2 , and 3 times $G_{0}$ or even higher have been seen in the case of $\mathrm{Au}$ (Refs. 10,26,19,14), Pt (Refs. 30,26), and Cu (Refs. 26,19) at room temperature. In Fig. 2 we show the histogram based on 227 indentations on a $\mathrm{Au}(110)$ surface (see Ref. 10 for further details). For $\mathrm{Na}$, peaks in the histogram have been seen at 1,3 , and less clear peaks at 5 and 6 times $G_{0}$ at low temperature. ${ }^{18}$ These latter findings can be explained based on the degeneracy of the quantum modes if the neck in the case of $\mathrm{Na}$ is preferentially cylindrical.

The appearance of integer multiples of the conductance unit $G_{0}$ strongly suggests the concept of well-defined quantum modes in the contacts as a starting point. However, the abrupt atomic rearrangements definitely also play a role. Combined force and conductance measurements during the tip pulloff shows that the drop in adhesive force is correlated with a drop in conductance. ${ }^{13}$ These observations are in quantitative agreement with the prediction in Ref. 10. The picture that emerges is one in which the quantized conductance at integer values of $G_{0}$ is determined by the quantum modes but the jump between the different values are caused by the abrupt atomic rearrangements. However, there are also exceptions to this scenario. In a situation where the cross sectional area of a contact is varied smoothly the quantum modes could still give rise to quantized conductance but the transition between the different steps could be expected to be less abrupt. A more smooth transition between steps is sometimes seen at a low temperature using the high stability MCB setup, as shown in the first curve for $\mathrm{Cu}$ and the last curve for $\mathrm{Na}$ in Fig. 3. At higher temperatures and in air the steps for Au observed with STM are abrupt and accompanied by large steps in the force. ${ }^{13} \mathrm{~A}$ special behavior is seen for the semimetallic antimony ( $\mathrm{Sb})$. With its low conduction electron density, the Fermi wavelength is about an order of magnitude larger than for the other metals studied ( $\lambda_{F} \approx 110$ bohr). During the pull of an Sb contact steps are 


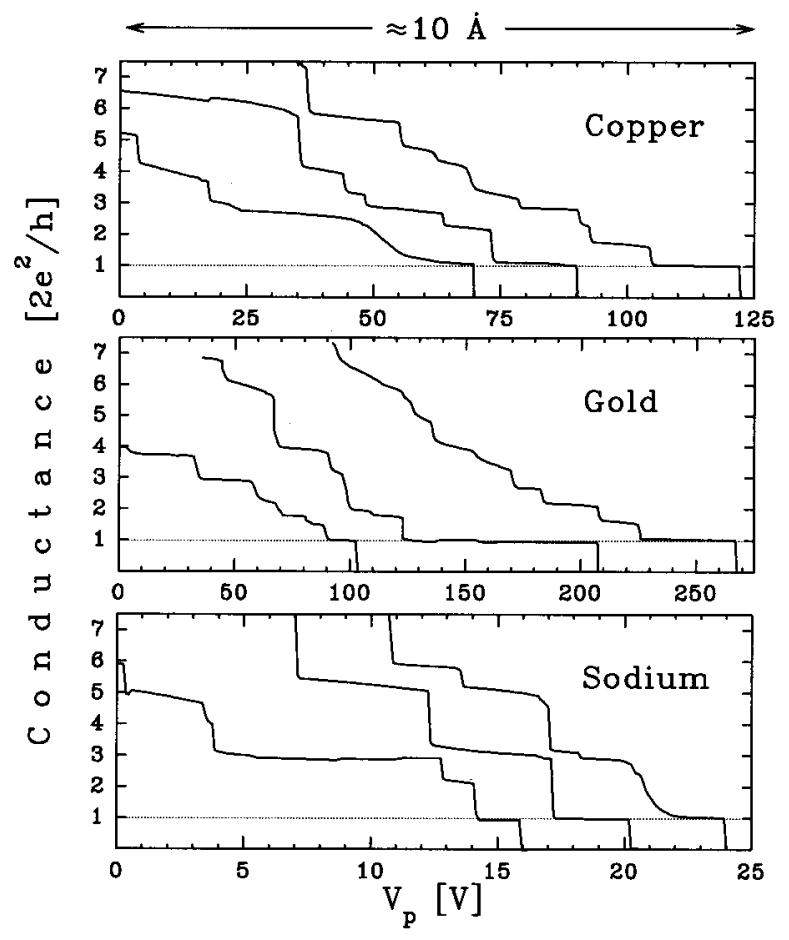

FIG. 3. Conductance curves obtained using the MCB technique at helium temperatures (Ref. 20).

observed ${ }^{17}$ which are a small fraction of $G_{0}$. These steps can only result from atomic rearrangements in the contact. For bigger $\mathrm{Sb}$ contacts the conductance is not found to be quantized possibly because these big contacts do not have an elongated shape, especially at cryogenic temperatures. ${ }^{20}$

In the present paper we report an exact calculation of the electronic transport through idealized small contacts in the presence of elastic scattering. We investigate the stability of the quantum conductance steps when the neck structure deviates from an ideal one with well-defined quantum modes. Especially, we will see how different scattering sources, point scatterers located inside the neck and boundary corrugation, will influence the conductance. We will use a jelliumlike model which is known to describe metals like $\mathrm{Na}$ or the noble metals quite well, as has been seen in the case of models for small metal clusters. ${ }^{31}$ We find that a localized scatterer will smear and downshift the quantum steps selectively depending on its location. In the case of boundary corrugation steps can be substantially downshifted without suffering from severe smearing depending on the length scale of the corrugation. We find that the quantum mode picture, in general, is quite stable and that the shifted conductance steps still can be assigned to a particular quantum mode.

The paper is organized as follows. In the first section we will shortly review results obtained from simulations of neck structures to get an atomistic picture of the structures involved. Then the concepts of mesoscopic transport theory are introduced and in this framework the quantized conductance is discussed. This is followed in Sec. III by a short discussion of models used for calculations of transmission through 3D constrictions and an outline of the recursion method used for the computations in this paper. In Sec. IV the results of the computations are presented and discussed.

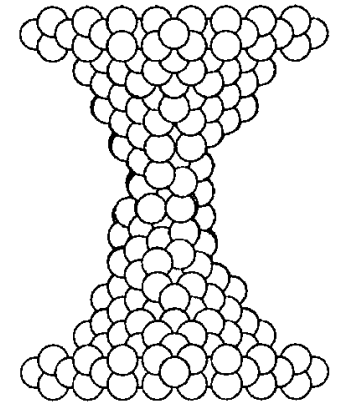

FIG. 4. Snapshot from computer simulation of neck formation.

\section{ATOMIC SCALE METAL CONTACTS AND CONDUCTANCE QUANTIZATION}

\section{A. Simulations of contacts}

Atomistic molecular dynamics (MD) simulations of the dynamics of the contact breaking process has been carried out for many different metals: Ni tip and Au surface, ${ }^{32} \mathrm{~Pb}$ tip on a $\mathrm{Pb}$ surface with or without a Ir layer and a $\mathrm{Ni}$ tip on a Ag substrate, ${ }^{33}$ Ir tip and surface, ${ }^{34} \mathrm{Ni}$ tip and surface, ${ }^{35} \mathrm{Cu}$ tip and surface, ${ }^{10}$ and also for Au nanowires. ${ }^{10,27}$ The formation of a "neck" (see Fig. 4) is seen in all the simulations and the evolution of the neck during the stretch of the contact is quite similar for the contacts. The cross-sectional area decreases slowly, while the adhesive force is built up by elastic deformation. This goes on until a sudden restructuring of the neck occurs. During the restructuring, the cross-sectional area changes along with a rapid drop in the adhesive force resulting in a thinner and longer neck. In the simulations the starting point has been crystalline tip structures with well defined atomic layers. The restructuring in the first part of the stretch is accomplished by emission of dislocations while in the later part the restructuring goes on via a highly disordered stage where the layers in the vicinity of the narrowest part of the contact cannot be distinguished. Landman et al. ${ }^{27}$ have simulated the process of elongation followed by compression of a nanowire and compared with experiments. They find that the process to a large extend is reversible for the considered size of wires (conductances higher than about $\left.10 G_{0}\right)$. It has been pointed out by Bratkovsky, Sutton and Todorov $^{35}$ that the temperature critically influences the reordering processes because these depend on the available kinetic energy to initiate atomic rearrangements.

A severe problem is that only a short time is available in the MD simulations $(\sim 0.1 \mathrm{~ns})$ compared to the typical experimental time scale $(\sim 1 \mathrm{~ms})$. This means that the very temperature sensitive diffusion processes cannot be included in the MD simulations. In experiments on $\mathrm{Cu}$ (Ref. 19) the conductance quantization is only prominent at high temperatures, whereas in the case of Au (Ref. 14) no significant dependence of temperature is seen.

\section{B. Conductance quantization}

In the simulations it is, in general, seen that the contacts have their main variation in shape on a length scale of several atomic interlayer distances due to elastic deformation, see Fig. 4. The one-electron potential therefore has an envelope with a curvature on this length scale superimposed by a 

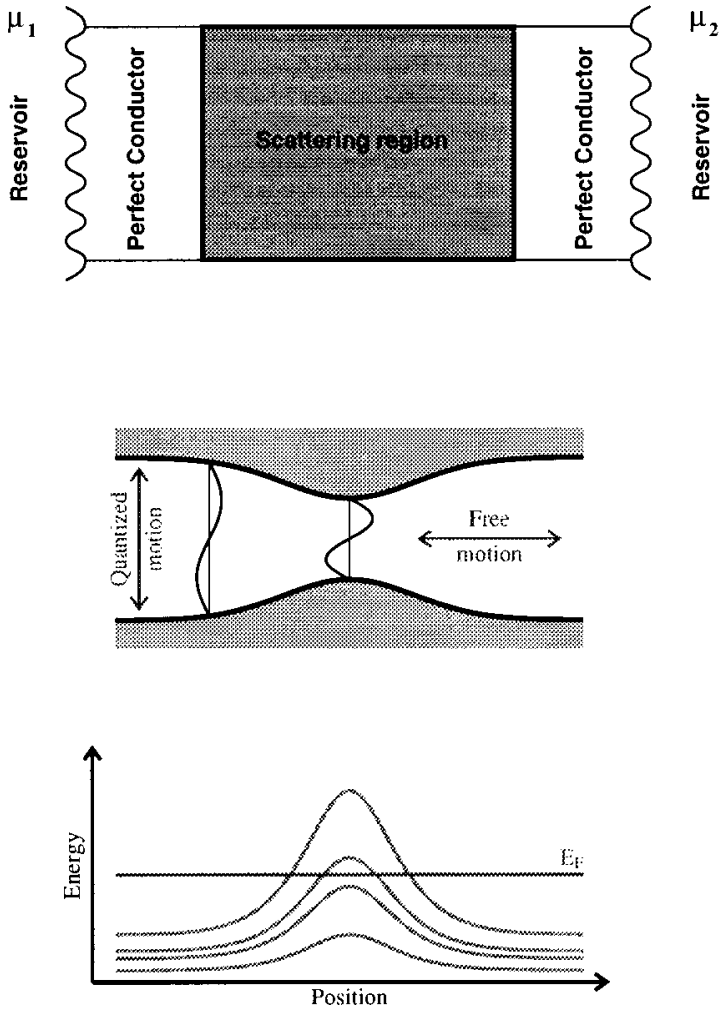

FIG. 5. Upper: The setup where the Landauer formula for ballistic quantum transport applies. Only elastic scattering processes are assumed to occur in the scattering region (all inelastic processes take place in the reservoirs), no scattering takes place in the perfect conductors and between the perfect conductors and the reservoirs. Middle: In the case of slowly varying confining potential the adiabatic principle applies. Lower: To each adiabatic transverse mode corresponds a potential barrier in the longitudinal direction due to the energy taken up by the transverse motion. The finite number of modes with a barrier less than $E_{F}$ contributes each with the conductance quantum $2 e^{2} / h$.

smaller atomic corrugation from the surface atoms. On top of this comes internal potential variations, especially in the case of internal disorder with large variations in interatomic bond lengths, and locally big variations in the boundary corrugation due to a single or a few atoms in unfavorable positions.

The starting point for the understanding of the conductance quantization in the atomic scale metal contacts is the smooth potential with an envelope varying on the length scale of several interlayer distances. With this as the starting point the quantized conductance can be understood in terms of the Landauer's ${ }^{36-38}$ formula of quantum ballistic transport combined with the adiabatic ${ }^{39,40}$ principle.

The Landauer formula is based on the assumptions ${ }^{37,38}$ that the system can be viewed as a scattering region where solely elastic scattering takes place, connected to two perfect leads (no scattering) which define incoming and outgoing states. These are then connected to two electron reservoirs at infinitesimally different chemical potentials. This setup is depicted in Fig. 5. The effect of the reservoirs is to fill the states in the perfect leads to the chemical potential of the reservoir, according to the Fermi-Dirac distribution. It is assumed that electrons entering the reservoirs from the perfect leads are not reflected at the joining, and that the electrons entering the reservoirs are not influencing the reservoir populations. The conductance, $G$, is then determined by the elastic amplitudes, $t_{i j}$, for transmission from the propagating incoming state $i$ in the first perfect lead to the propagating outgoing state $j$ in the second lead at the Fermi energy,

$$
G=\frac{2 e^{2}}{h} \sum_{i j}\left|t_{i j}\left(E_{F}\right)\right|^{2} \text {. }
$$

The adiabatic principle is applied in the same sense as in a molecule where the Schrödinger equation separates into one part for the electrons and one for the positions of the ions which only appear as parameters in the electronic part. Here the fast transverse quantized motion acts as the electrons while the slow variation of the contact shape acts as the ions. In the adiabatic case the transverse modes will not mix, and the corresponding quantum numbers will be conserved all the way from the first perfect lead to the second. This conductance channel will experience an effective potential due to the energy tied up in the transverse motion along the way, see Fig. 5.

As an example, we could consider a constriction with rotational symmetry around the axis in the direction of propagation (this will be $z$ in the following) and with a hardwall boundary at radius $R(z)$ in the $x y$ direction. The wave functions are expanded (for each $z$ ) in terms of the transverse modes $\Phi_{n m z}(r, \varphi)$,

$$
\begin{gathered}
\Psi(z, r, \varphi)=\sum_{(n m)} c_{n m}(z) \Phi_{n m z}(r, \varphi), \\
\Phi_{n m z}(z, \varphi)=\frac{1}{J_{m+1}\left(\alpha_{n m}\right)} J_{m}\left(\alpha_{n m} \frac{r}{R(z)}\right) e^{i m \varphi},
\end{gathered}
$$

where $J_{m}$ is the $m$ th Bessel function with $n$th root $\alpha_{n m}$. The Schrödinger equation expressed in this basis is

$$
\begin{aligned}
& {\left[-\frac{\hbar^{2}}{2 m} \frac{\partial^{2}}{\partial z^{2}}+\varepsilon_{n m}(z)-E\right] c_{n m}(z),} \\
& \quad+\sum_{\left(n^{\prime} m^{\prime}\right)} U_{n m, n^{\prime} m^{\prime}}(z) c_{n^{\prime} m^{\prime}}(z)=0,
\end{aligned}
$$

where

$$
\varepsilon_{n m}(z)=\frac{\hbar^{2}}{2 m}\left(\frac{\alpha_{n m}}{R(z)}\right)^{2} .
$$

Here $U$ depends on $d R / d z$ and $d^{2} R / d z^{2}$ and couples the different "channels"'/modes with quantum numbers $(n, m)$. In the adiabatic approximation this coupling is neglected and the electron transport takes place in each "channel" in parallel. Each mode encounter an effective 1D potential barrier, $\varepsilon_{n m}(z)$, which in the strict adiabatic case will be much broader than $\lambda_{F}$ and therefore either completely reflect or transmit, so the Landauer two-terminal formula is in that case simply

$$
G=\frac{2 e^{2}}{h} \sum_{(n m)} T_{n m}\left(E_{F}\right),
$$

where $T_{n m}$ is the corresponding transmission probability (either 0 or 1). This is the origin of the quantum step structure, 
because as the height of the effective barrier (the width of the constriction) is reduced, channels will close, changing the conductance by the quantum unit times the degeneracy of the closing channel. We have taken the limit of vanishing temperature since the energy scales involved in the transmission (differences between transverse modes $\sim 1 \mathrm{eV}$ ) are much larger than $k T$ in the $3 \mathrm{D}$ metal contact experiments.

The general criterion ${ }^{39,40}$ for adiabatic transport is $d R / d z \ll 1 / N_{a}(z), N_{a}(z)$ being the local number of available transverse modes above the barrier. Real potentials will, in general, display nonadiabaticity: The width usually grows faster towards the end of the constriction potential, while the local number of transverse modes is growing. However, the adiabatic condition does not have to be satisfied globally. Yacoby and Imry have shown ${ }^{40}$ that the reflections due to a sudden opening is highly suppressed if there is a small adiabatic widening preceding it. The local reflection amplitudes scale with $\left(1 / N_{a}(z)\right) d R / d z$ explaining the strongly reduced backscattering when $N_{a}$ grows towards the opening of the constriction. They have also shown that the corrections to the adiabatic picture is of the same order as the scattering by the $1 \mathrm{D}$ effective potential, i.e., the tunneling and reflection above the barrier. This was used in Ref. 10 to estimate the scattering from the boundary neglecting interchannel scattering. It has been shown in the $2 \mathrm{D}$ case by Maab et al. ${ }^{41}$ that the neglect of the coupling terms between channels underestimates the degree of quantization.

\section{CONDUCTANCE CALCULATIONS}

We shall first briefly review some earlier works on transmission through restricted geometries in 3D. Then the computational method used in this paper is introduced.

\section{A. Constriction models and transmission}

Exact analytical expressions for the 3D transmission has been obtained for simplified geometries. Bogachek et al. ${ }^{42}$ discussed the case of a hard-wall cone-type rotational symmetric modelpotential. Extending a hyperbolic hard-wall constriction used by Yosefin and Kaveh ${ }^{43}$ in 2D to the corresponding 3D case with cylindrical symmetry, Torres et al. ${ }^{44}$ calculated exactly the transmission as a function of width for different opening angles. They show how the conductance steps get more smeared when the opening angle is made larger, approaching the semiclassical limit,

$$
G_{\infty}=G_{0}\left(\frac{k_{F}^{2} A}{4 \pi}-\frac{k_{F} P}{4 \pi}\right) .
$$

This is the Sharvin ${ }^{45}$ formula for ballistic transport with a perimeter $(P)$ correction. For a square hard-wall confining potential with the transverse modes $\sin \left(n_{x} x \pi /\right.$ $L) \sin \left(n_{y} y \pi / L_{y}\right)$, this correction subtracts the modes where $n_{x}$ or $n_{y}$ are zero. Scherbakov et al. ${ }^{46}$ have investigated the quantum step structure in a free electronlike model in the presence of a magnetic field. They describe the neck potential by a harmonic saddle point $\left(\propto-k_{z} z^{2}+k_{x} x^{2}+\right.$ $k_{y} y^{2}$ ), for which the transmission is exactly known as a function of $E_{F}$, which is the quantity varied in the paper. In this model the degeneracy of the quantized steps due to the degeneracy of transverse modes may be lifted by choosing $k_{x}$ $\neq k_{y}$. However, the shapes of the necks will, in general, be elongated, which is known to improve the conductance quantization. Many numerical investigations has been carried out. Torres and Sáenz ${ }^{47,48}$ have modeled the elongated structures by stacking cylindrical layers with a thickness of the atomic interlayer spacing and varying radii. The electrons were confined in these by a hard-wall potential. The transmissions were calculated using a generalized scattering-matrix technique to match the modes between layers. Kasai et al. ${ }^{49}$ have modeled the 3D constriction using a tight-binding model varying the on-site energies.

In a number of studies atomic structures obtained with molecular dynamics simulations have formed the basis for simple conductance calculations. ${ }^{34,35,10}$ The conductance calculations have then been carried out in either free-electronlike models with a hard-wall constriction ${ }^{35,10}$ or using a tightbinding approximation. ${ }^{34,35}$ We would like here to point out an important difference between the free-electron-like models and the tight-binding model if - as is often the case a very limited basis set is used in the tight-binding description. The systems we have in mind in this context are, for example, alkali metals or noble metals where a free-electronlike model is known to describe the states at the Fermi level of the bulk metals reasonably well. The difference between the free-electron model and the tight-binding model can be seen already by considering the local density of states in a region of atomic size. In the case of the free-electron gas the density of states is increasing with energy with a square root dependence but for a tight-binding model with, say, a single $s$ state per atom, the density of states will have a high value around the energy of the orbital and then decrease at higher energies. The free electron gas can of course be well described in a given energy window using an atomic tightbinding basis, but the energy overlap and the hybridization between different bands $(s, p, d \ldots)$ will generally be considerable and several bands will be necessary to describe the states near the Fermi level in an appropriate way. This point also carries over to the situation of transmission through an atomic size neck. If we for simplicity consider transmission through a single atom and only include one state on this atom we obtain a resonant tunneling description: The transmission will go through a maximum value near the position of the state, $\varepsilon_{a}$ (properly renormalized by the leads), and at higher energies the transmission falls off. The conductance is (see, e.g., Ref. 50),

$$
G=G_{0} \frac{4 \Delta_{L} \Delta_{R}}{\left(E_{F}-\varepsilon_{a}\right)^{2}+\left(\Delta_{L}+\Delta_{R}\right)^{2}},
$$

where the width of the transmission peak is determined by the strength of the coupling between the atom and the leads ( $L$ and $R$ ) expressed in terms of the coupling weighted density of states ${ }^{51}$ of the leads (assumed to be independent of energy, wide band limit). This is a description appropriate for a situation with weak coupling to the leads. However, if this coupling is sufficiently strong, so that the width of the transmission peak is comparable to the energy difference between atomic states, the true transmission will not decrease at higher energies but increase until saturation is reached.

In this one-state case the maximum of $1 G_{0}$ is reached for $E_{F}=\varepsilon_{a}$ and $\Delta_{L}=\Delta_{R}$. In the case of more sites connecting 
the electrodes and when only hopping can occur between neighboring atomic layers, the conductance will be limited to the minimum number of sites/states in a cross section of the neck times $G_{0}$. This indicates that the scattering of a propagating wave in the tight-binding model is much more severe than in the free-electron model due to the limited basis set. The sensitivity of the hopping matrix elements on the interatomic distances will be reflected in the conductance, because inhomogeneities in the hopping will increase scattering and give deviations from the quantized values. This can be seen crudely in the one-state case where different bond lengths to the two electrodes will make $\Delta_{L}$ different from $\Delta_{R}$ and the maximum conductance less than $G_{0}$.

The weakness of the simple free-electron-like models is that scattering due to internal and boundary disorder is not taken into account. These features are naturally accounted for in the tight-binding models without further complications, whereas a larger numerical effort is required for the free-electron models. We will use here a model potential with free-electron electrodes as our starting point and include the scattering effects in the neck structure using an exact numerical computational scheme.

\section{B. Computational method}

Now we shall discuss the numerical method which we have used to calculate the transmission in our jellium like model where scattering from the individual atomic pseudopotentials are neglected. We have employed the recursiontransfer-matrix method of Hirose and Tsukada. ${ }^{52}$ This method is an extension of the 1D continued fraction/recursion method of Lambin and Vigneron ${ }^{53,54}$ to the 3D multichannel case.

For a numerical calculation of the stationary solutions to Schrödingers equation with scattering boundary conditions and getting the transmission, we will have to discretize the problem. This is done in two steps. The first step is to replace the single neck-structure along the $z$ direction by a periodically repeated unit cell in the $x y$ direction each containing the neck structure. This allow us to use Bloch's theorem and the $x y$ part of the problem can be written in terms of the discrete reciprocal lattice vectors $\left(\vec{G}_{\perp}^{j}\right)$ corresponding to the translational symmetry in the $x y$ plane. The $i$ th stationary scattering state can then be written as

$$
\psi_{i}\left(z, \vec{r}_{\perp}\right)=e^{i \vec{k}_{\perp} \cdot \vec{r}_{\perp}} \sum_{j} \phi_{i j}(z) e^{i \vec{G}_{\perp}^{j} \cdot \vec{r}_{\perp}}
$$

where $\vec{k}_{\perp}$ is the conserved Bloch $k$ vector in the $x y$ Brillouin zone. Since it is conserved we will take it to be implicit in the rest. The $\vec{G}_{\perp}^{j}$ in this basis define the conductance "channels." However, the plane-wave basis set is very far from the adiabatic basis set in the sense that the different $\vec{G}_{\perp}^{j}$ channels will mix strongly inside the neck structure. The advantage is that they will be decoupled in the free-electron electrodes. Now, we have obtained discrete channels, however, we still have an infinite number of them and we will have to truncate the $\vec{G}_{\perp}$ set. This is done by including only the set of $\vec{G}_{\perp}$ within the energy cutoff sphere:

$$
\left|\vec{k}_{\perp}+\vec{G}_{\perp}^{i}\right|^{2}<\left(2 m E_{\text {cut }} / \hbar^{2}\right),
$$

and the numerical calculations will then have to be converged with respect to the cutoff energy, $E_{\text {cut }}$.

The next step is to calculate the transmission through one unit cell which, for a sufficiently big unit cell, will converge to the transmission in the non-periodic potential. This is done by a real-space discretization along the $z$ direction. Defining the coefficient matrix $\underline{\underline{\Psi}}$,

$$
(\underline{\underline{\Psi}}(z))_{i j} \equiv \phi_{i j}(z),
$$

the Schrödinger equation can be written in matrix form as

$$
-\frac{\hbar^{2}}{2 m} \frac{\partial^{2}}{\partial z^{2}} \underline{\underline{\Psi}}(z)+\underline{\underline{V}}(z) \underline{\underline{\Psi}}(z)=E \underline{\underline{\Psi}}(z),
$$

with scattering boundary conditions,

$$
(\underline{\underline{\Psi}}(z))_{i j}= \begin{cases}t_{i j} e^{i k_{z}^{j} z} ; & z \text { in exit } \\ \delta_{i j} e^{i k_{z}^{i} z}+r_{i j} e^{-i k_{z}^{j} z} ; & z \text { in entrance }\end{cases}
$$

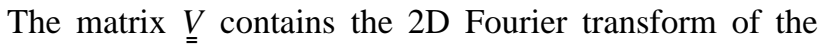
potential and the kinetic energy in the $x y$ plane,

$$
\begin{aligned}
(\underline{\underline{V}}(z))_{i j}= & \delta_{i j} \frac{\hbar^{2}}{2 m}\left|\vec{k}_{\perp}+\vec{G}_{\perp}^{i}\right|^{2} \\
& +\frac{1}{A_{\text {u.c. }}} \int_{\text {u.c. }} V\left(\vec{r}_{\perp}, z\right) e^{i\left(\vec{G}_{\perp}^{j}-\vec{G}_{\perp}^{i}\right) \cdot \vec{r}_{\perp} d \vec{r}_{\perp},}
\end{aligned}
$$

where $A_{\text {u.c. }}$ is the $x y$ area of the unit cell. The Schrödinger equation is discretized using the Numerov method which is correct to 4 . order in the $z$ grid division, $h_{z}$ :

$$
\underline{\underline{a}}\left(z_{p}\right) \underline{\underline{\Psi}}\left(z_{p+1}\right)-\underline{\underline{b}}\left(z_{p}\right) \underline{\underline{\Psi}}\left(z_{p}\right)+\underline{\underline{c}}\left(z_{p}\right) \underline{\underline{\Psi}}\left(z_{p-1}\right)=0,
$$

where

$$
\begin{gathered}
\underline{\underline{a}}\left(z_{p}\right)=\underline{\underline{I}}-\frac{1}{12} \frac{2 m h_{z}^{2}}{\hbar^{2}}\left[\underline{\underline{V}}\left(z_{p+1}\right)-\underline{\underline{I}} E\right], \\
\underline{\underline{b}}\left(z_{p}\right)=2 \underline{\underline{I}}+\frac{5}{6} \frac{2 m h_{z}^{2}}{\hbar^{2}}\left[\underline{\underline{V}}\left(z_{p}\right)-\underline{\underline{I}} E\right], \\
\underline{\underline{c}}\left(z_{p}\right)=\underline{\underline{a}}\left(z_{p-2}\right) .
\end{gathered}
$$

The trick in the recursion method ${ }^{53}$ is to consider the ratio corresponding to the logarithmic derivative of the wave function, i.e., the phase,

$$
\underline{\underline{S}}\left(z_{p}\right)=\underline{\underline{\Psi}}\left(z_{p+1}\right) \underline{\underline{\Psi}}\left(z_{p}\right)^{-1} .
$$

The reason is that $\underline{\underline{S}}$ is known on the exit side due to the boundary conditions (13), since the unknown $t_{i j}$ cancels out in $\underline{\underline{S}}$ there (while $r_{i j}$ will not cancel out in the entrance). Taking the potential in the free-electron electrodes to be zero, we have the solution to Eq. (15) in the electrodes,

$$
\underline{\underline{S}}=\underline{\underline{K}},
$$

where 


$$
(\underline{\underline{K}})_{i j}=\delta_{i j}\left(\gamma_{i}+i \sqrt{1-\gamma_{i}^{2}}\right)
$$

with

$$
\gamma_{i}=\frac{1+\frac{5}{12} h_{z}^{2}\left[\left|\vec{k}_{\perp}+\vec{G}_{\perp}^{i}\right|^{2}-\left(2 m E / \hbar^{2}\right)\right]}{1-\frac{1}{12} h_{z}^{2}\left[\left|\vec{k}_{\perp}+\vec{G}_{\perp}^{i}\right|^{2}-\left(2 m E / \hbar^{2}\right)\right]} .
$$

$\underline{\underline{K}}$ is just the discretization of the phase,

$$
\delta_{i j} e^{i h_{z} \sqrt{\left(2 m E / \hbar^{2}\right)-\left|\vec{k}_{\perp}+\vec{G}_{\perp}^{i}\right|^{2}}} .
$$

From Eq. (15) a one point recursion relation for $\underline{\underline{S}}$ is obtained,

$$
\underline{\underline{S}}\left(z_{p-1}\right)=\left[\underline{\underline{b}}\left(z_{p}\right)-\underline{\underline{a}}\left(z_{p}\right) \underline{\underline{S}}\left(z_{p}\right)\right]^{-1} \underline{\underline{c}}\left(z_{p}\right) .
$$

The recursion can then be started in the exit electrode and yield $\underline{\underline{S}}$ in the entrance,

$$
\underline{\underline{S}}\left(z_{-1}\right)=\underline{\underline{\Psi}}\left(z_{0}\right) \underline{\underline{\Psi}}\left(z_{-1}\right)^{-1} .
$$

Using the boundary condition for the entrance $[$ Eq. (13)] $\underline{\underline{\Psi}}$ can be written here as

$$
\underline{\underline{\Psi}}\left(z_{p}\right)=\underline{\underline{K}}^{p}+\underline{\underline{K}}^{-p} \underline{\underline{\underline{r}}} .
$$

From the last two equations, the unknown reflection amplitude matrix can be eliminated, and we can obtain the coefficient matrix in the entrance,

$$
\underline{\underline{\Psi}}\left(z_{0}\right)=\underline{\underline{S}}\left(z_{-1}\right)\left[\underline{\underline{K}} \underline{\underline{S}}\left(z_{-1}\right)-\underline{\underline{I}}\right]^{-1}\left[\underline{\underline{K}}-\underline{\underline{K}}^{-1}\right],
$$

and from this the transmission amplitude matrix, $\underline{\underline{t}}$, can be calculated:

$$
\underline{\underline{t}}=\underline{\underline{\Psi}}\left(z_{N}\right)=\prod_{p=0}^{p=N} \underline{\underline{S}}\left(z_{p}\right) \underline{\underline{\Psi}}\left(z_{0}\right) .
$$

The transmission amplitude matrix that we have obtained gives us the amplitude for entering in "channel" $i$, i.e., in a plane wave with a transverse wave vector $\vec{G}_{\perp}^{i}\left(+\vec{k}_{\perp}\right)$, and exiting in "channel" $j$ in a plane wave with a transverse wavevector $\vec{G}_{\perp}^{j}\left(+\vec{k}_{\perp}\right)$. To get the flux-normalized $t_{i j}$ entering the Landauer formula, we will have to multiply our obtained $t_{i j}$ by $\sqrt{k_{z}^{j} / k_{z}^{i}}$.

The recursion calculation of the transmission must be performed for a sufficiently large set of $\vec{k}_{\perp}$ vectors and the average of conductances must be taken in the $x y$ Brillouin zone. For a sufficiently large $x y$-unit cell it will be sufficient to use only $\vec{k}_{\perp}=0$. In the numerical calculations the computing time is mainly spend on 2D Fourier transforms and matrix inversions which can be performed efficiently by standard computer library routines.

\section{RESULTS}

In this section we present the results of our calculations using the recursion method. We consider electrons with $E_{F}=5.5 \mathrm{eV}$ and a soft-wall confining potential of height $2 \times E_{F}$, as a simplified version of a Au-jellium model. In all calculations we have used a unit cell of $60 \mathrm{bohr}$ in the $x$ and $y$ directions. The $x y$ grid used in the numerical Fourier transforms have $60 \times 60$ points and we use a recursion step length

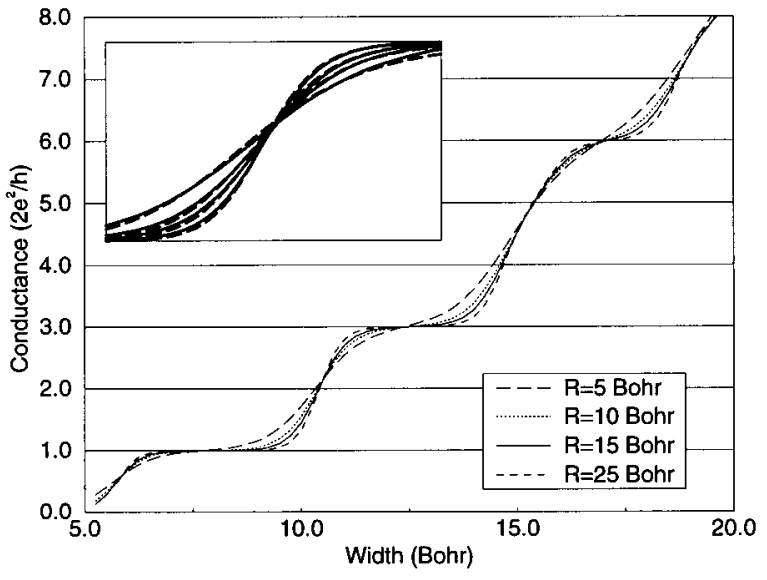

FIG. 6. Conductance curves for circular shaped envelopes with different radii of curvature $(R)$. The cross section is chosen to be circular. In the inset the $1 G_{0}-3 G_{0}$ step (solid) is plotted together with the approximate expression (dashed).

in the $z$ direction of 1 bohr. The conductance curves are converged for a plane-wave cutoff of $20.5 \mathrm{eV}$ corresponding to 408 channels.

\section{A. Smooth 3D contacts}

We have here chosen to consider the simplest possible shapes even though the method is capable of treating general shapes. The reason for this is that we want to focus primarily on the effects originating from the scattering which is not intrinsic to the smooth envelope. Also for simplicity we shall not take into account the change of shape of the neck during the elongation but just consider the width of the neck as the only variable.

We define our neck shapes in the following way:

$$
V(r, z)=V_{0}\left\{\begin{array}{c}
\text { For } r>R+g W / 2: \theta(R+z) \theta(R-z) \\
\quad \text { otherwise } \\
\theta\left\{R-\sqrt{z^{2}+[r+(R+g \cdot W / 2)]^{2}}\right\}
\end{array}\right.
$$

where

$$
r=g \sqrt{(x / \alpha)^{2}+(\alpha y)^{2}} .
$$

For $g=1, R$ is the radius of curvature of the circular neck envelope in the $r z$ plane and $W$ is the "width" of the neck at the thinnest point. We can deform this shape from circular $(g=1)$ to elliptical $(g>1)$. The cross section can be deformed from a circle $(\alpha=1)$ to an ellipse with axis lengths $r_{x}=\alpha W$ and $r_{y}=W / \alpha$. Instead of step functions ( $\theta$ ) we use Fermi functions with a width of 0.5 bohr.

In Fig. 6 we show the conductance curves resulting from necks with circular cross sections $(\alpha=1)$ and circular envelopes $(g=1)$. The curves correspond to four different radii of curvature of the envelope, $R$. It is seen how the smearing of the steps gets stronger with decreasing $R$. This is illustrated in Fig. 7, where we plot the contours in the $x z$ plane (cut through the middle of the neck in the direction of the current) of the $z$ component of the current density, $j_{z}$. In the left panel we see the current pattern for $R=5 \mathrm{bohr}$ (upper) 

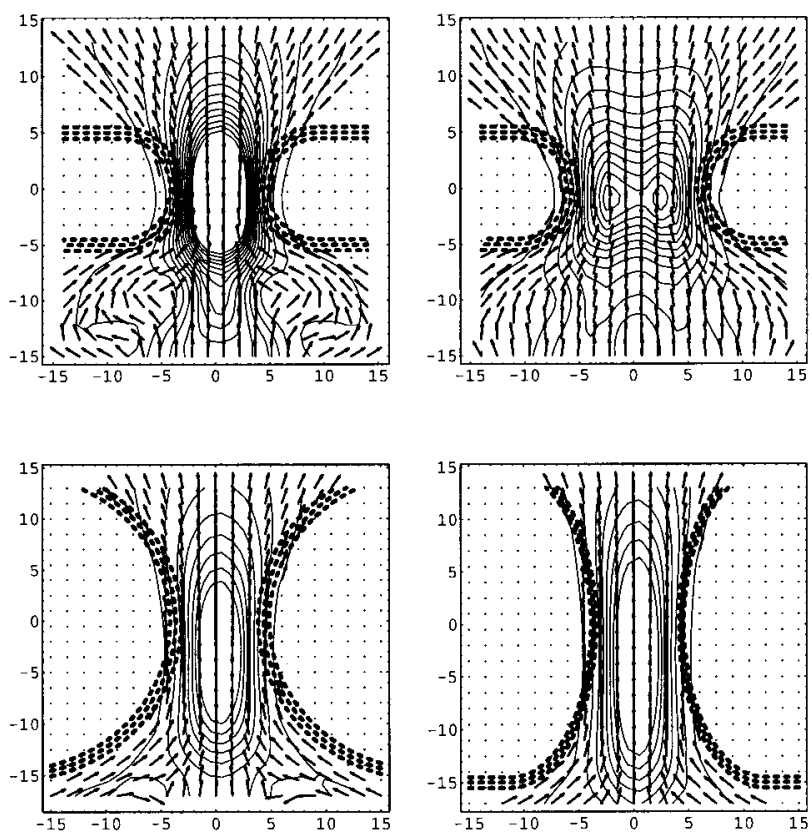

FIG. 7. Top panel: Contours of current density in $z$ direction, $j_{z}$, and the direction of current for a highly curved constriction, $R=5 \mathrm{bohr}$, for widths of $8 \mathrm{bohr}$ (left) and $12 \mathrm{bohr}$ (right). The left contours values are multiplied by 0.5 compared to right. The corresponding conductances are $1.01 G_{0}$ and $2.92 G_{0}$, respectively. The constricting potential is shown by dashed contour lines (contours: $\left.E_{F} \pm 0.25 E_{F}\right)$. Note the peak structure inside the neck. Lower panel: The contours for a less curved constrictions with $R=15$ bohr, spherical $g=1$ (left) and elliptical $g=2$ (right) shapes. The conductance is in both cases $1.0 G_{0}$.

and $R=15$ Bohr (lower) for a width corresponding to a conductance of $1 G_{0}$. The more symmetric pattern in the latter case indicates less backscattering. In the upper right panel we show the pattern for $R=5$ and a width corresponding to a conductance of $2.9 G_{0}$. Here two maxima in the current density are seen inside the constriction. In the $2 \mathrm{D}$ calculations with flat constriction sides it has been pointed out ${ }^{55}$ that an accurate quantization persist even for constrictions with lengths much shorter than the width.

Let us consider the step shape of the opening of a single conductance step corresponding to a conductance of $G=$ $N G_{0}$ and with degeneracy $n_{\mathrm{deg}}$, as a function of the neck width $(W)$. If we approximate this by a Fermi function,

$$
\begin{gathered}
\delta G(W) \approx G_{0} n_{\operatorname{deg}} \frac{1}{1+e^{-\gamma(W) / \Delta}}, \\
\gamma(W)=\left(\frac{k_{F}^{2} W^{2}}{16}-\frac{k_{F} W}{4}\right)-N,
\end{gathered}
$$

the smearing of the steps is within the adiabatic picture approximately given by ${ }^{10}$

$$
\Delta \sim \frac{1}{2 \pi^{2}} \frac{\left(\pi W^{2} / 4\right)^{0.75} k_{F}}{\sqrt{2 R_{\mathrm{eff}}}} .
$$

Here $R_{\text {eff }}$ is the effective local curvature at the thinnest point. In the inset of Fig. 6 this formula is compared with the nu-

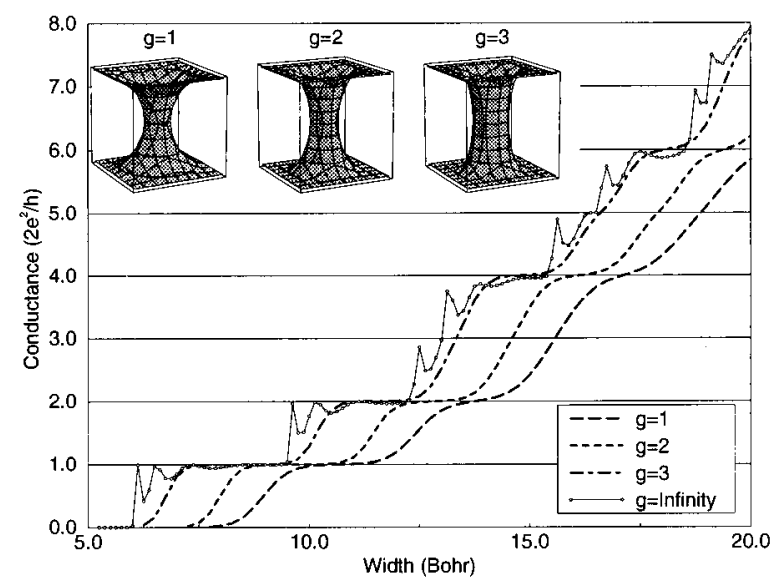

FIG. 8. The conductance curves for different elliptical shapes (inset) denoted by $g$. Here $R=15$ bohr and the cross section is taken to have $\alpha=0.75$ eccentricity. Now steps $G=2 G_{0}$ and $G=4 G_{0}$ are seen, but $G=3 G_{0}$ has disappeared. In the case of a cylindrical hole connecting the electrodes $(g=\infty)$ a resonance structure is seen. The curves have been shifted $(0,1,2,3$ bohr) horizontally for clarity.

merical exact result for the $1-3 G_{0}$ double step using $R_{\text {eff }}=R$. It is seen that the simple formula describes the scaling of the step with $R$ reasonably well.

Now we change the neck shape from a circular $(g=1)$ to an elliptical envelope $(g>1)$. The conductance curves are displayed in Fig. 8. We keep $R$ fixed at 15 bohr, i.e., the length of the neck is fixed at $2 R=30 \mathrm{bohr}$. In this calculation we have chosen the cross section to be elliptical with $\alpha=0.75$. This splits the degenerate modes corresponding to the $G=3 G_{0}$ and $G=5 G_{0}$ steps and steps at $2 G_{0}$ and $4 G_{0}$ appears instead (we will return to this point later). An increase in the quality of the quantization is clearly seen when the flatness $(g)$ of the neck side is increased. In the lower panel of Fig. 7 we compare the current pattern of the circular (left) with the $g=2$ elliptical (right) shaped envelope. The more flat $g=2$ gives a more focused current which is quite symmetric in exit and entrance, while the $g=1$ spreads the current and backscatters more into the entrance giving rise to the asymmetry. Within the adiabatic mode picture the more sharp transition between steps for $g=2$ compared to $g=1$ is understood in terms of less tunneling through and reflection above the effective barrier for the modes in the $g=2$ case. In the case of $g=\infty$, corresponding to a cylindric hole with no gradual opening, resonance structure is seen in the conductance curve. This is due to backscattering at the exit and entrance of the structure also seen in the 2D calculations. ${ }^{55-57,41}$ In the $3 \mathrm{D}$ case the flat sides with no gradual opening is quite unrealistic as seen from molecular dynamics simulations. Next we will turn to the effects of a single scatterer located inside an otherwise smooth constriction.

\section{B. Scattering center inside a smooth $3 D$ contact}

Localized scattering centers inside confined structures have been studied theoretically for the 2D systems. A $\delta$-function scatterer has been included in a constriction geometry $^{55}$ in a tight-binding calculation. Tekman and 


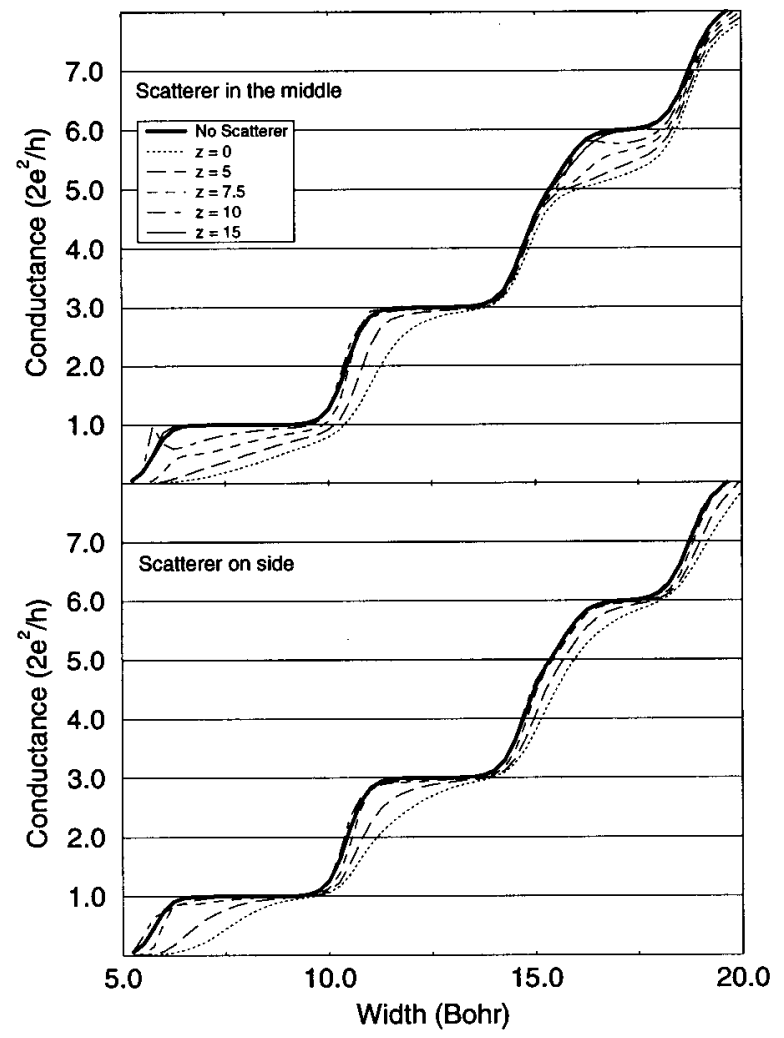

FIG. 9. Upper panel: The scatterer located in the middle of the neck ( $R=15$ bohr, $g=2)$ and moved along the $z$ axis towards the exit (entrance). It is seen that step 1 and step 6 are most affected. Lower panel: The scatterer located on the neck side. The step structure is maintained but smeared. The effect is reduced fast when moving the scatterer towards the exit (entrance) due to the curvature of the neck.

Ciraci $^{57}$ also considered a scatterer ( $\delta$ function in $z$ and finite extension in the transverse direction) in a $2 \mathrm{D}$ constriction geometry. Scatterers in infinite wires have been dealt with by Bagwell $^{58}$ who stresses the importance of the evanescent (tunneling) modes $\left(E_{n}>E_{F}\right)$ in the confined scattering problem. Recently Lang ${ }^{59}$ has calculated the conductance through three $\mathrm{Al}$ atoms in a line connected to jellium electrodes using a self-consistent scheme. Substituting one of the Al atom by a electro-negative sulfur atoms increases the resistance.

In these calculations we assume the following simple form for the change in the effective one-electron potential due to the presence of a scatterer located at $\overrightarrow{r_{0}}$ :

$$
V_{s}(\vec{r})=S \exp \left(-\left|\vec{r}-\vec{r}_{0}\right|^{2} / d^{2}\right) .
$$

We will refer to $S$ as the strength and $d$ as the decay length of the scatterer.

We choose a smooth elliptical constriction with shape $g=2, R=15$, and circular $x y$-cross section. The scattering potential is taken to be rather strongly repulsive with strength $S=2 E_{F}$ and a decay length of $d=2 \mathrm{bohr}$. In the upper panel of Fig. 9 conductance curves are shown for the scatterer placed in the middle of the neck at different $z$ positions. It is seen that, as expected, the effect is largest for the scatterer placed at $z=0$. It is also seen that step 1 and step 6 are almost completely "closed" in contrast to step 3, which re- covers quite fast, when moving the scatterer towards the neck opening. When the scatterer is placed close to the middle of the neck the electrons will have to tunnel and this gives an exponential dependence of the transmission on the width $(z=0$ bohr). Moving the scatterer towards the channel exit this goes into a more linear regime $(z=5$ bohr) and eventually a step less than $1 G_{0}$ appears $(z=7.5 \mathrm{bohr})$ which is followed by a slow increase towards $1 G_{0}$. We note that a resonance appears for $z=10$ for step 1 and is less clear for step 6 . This is presumably due to the reflections of the wave between the scatterer and the middle of the neck. We obtain the same results when the scatterer is placed symmetrically in the entrance $(z \rightarrow-z)$ due to the symmetric neck potential (the total transmission is always independent of the side of incidence).

The reason for the different influence of the scatterer on the different steps can be understood in a perturbation picture using the adiabatic states as a starting point. The change in conductance due to the extra backscattering can be estimated using the first Born approximation. Born approximation ${ }^{60}$ (and neglecting contributions from evanescent modes and the $z$ variation of the adiabatic wave functions within the range of the scattering potential $V_{s}$ ),

$$
\begin{aligned}
\delta G= & -\frac{2 e^{2}}{h} \sum_{n l}\left(\frac{m}{\hbar^{2}}\right)^{2} \frac{1}{k_{n} k_{l}} \\
& \times\left[\int_{-\infty}^{\infty} d z e^{i\left(k_{n}+k_{l}\right) z} \int_{-\infty}^{\infty} d x \int_{-\infty}^{\infty} d y\right. \\
& \left.\times \Phi_{l}(x, y) V_{s}(x, y, z) \Phi_{n}(x, y)\right]^{2},
\end{aligned}
$$

where $k_{n}(z)=\left\{2 m\left[E_{F}-\varepsilon_{n}(z)\right]\right\}^{1 / 2} / \hbar$ is the wave vector for the $n$th adiabatic mode. Here it is clear that for the $m$ th quantum conductance step, the dominant contributions to $\delta G$ comes from the modes with the smallest $k$, i.e., from the backscattering within the $m$ th adiabatic channel. These are simply the modes with the least energy left for propagation. Thus an adiabatic mode with a node where $V_{s}$ has its main weight will suffer less scattering. If we assume that the modes inside the neck resembles the modes in a hard-wall cylinder (see inset in Fig. 10), we see that the first and the sixth modes do not have a node in the middle like the other modes which have quantum number $m \neq 0$. It is also seen that modes 5 and 6 lie close in energy which explains why step 5 is smeared, because as 5 opens 6 will already begin to contribute to the conductance by tunneling.

To substantiate this point a little further, we have fixed the width of the neck at a given quantum step, 1, 3, or 6, and calculated the deviation from the step as the scatterer is moved from the middle of the neck to the side (along the $x$ axis). This is shown in Fig. 10. It is seen that the deviation as a function of scatterer displacement towards the side of the neck follows the structure of the cylinder wave functions for modes 1,3 , and 6 (see inset). If we now for simplicity assume a $\delta$ scattering potential,

$$
V_{s}(x, y, z)=S d^{3} \delta\left(x-x_{0}\right) \delta(y) \delta(z),
$$

and use the hard-wall potential wave functions in Eq. (35), we obtain 


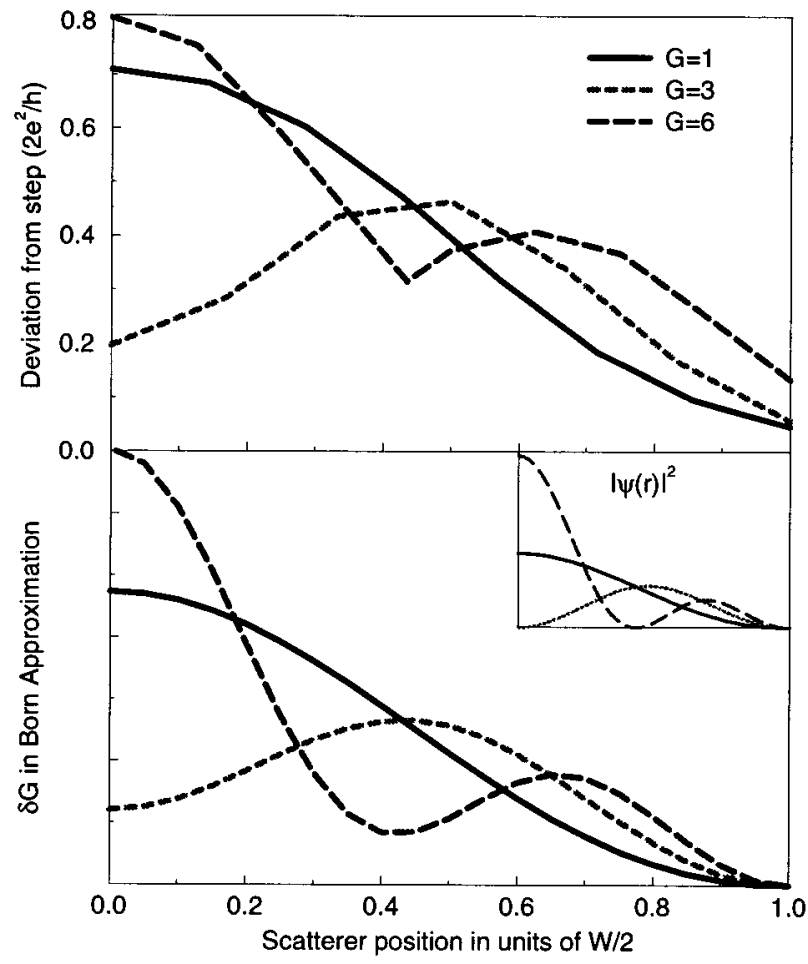

FIG. 10. The deviation from the integer quantum number is shown as a function of position of scatterer when this is moved from the middle to the side of the neck. This is shown for fixed widths corresponding to step $G=1 G_{0}, G=3 G_{0}$, and $G=6 G_{0}$, respectively. Below, result for deviation from integer quantum steps from Born approximation result using hard-wall cylinder wave functions [inset: Bessel functions, $J_{m}\left(\alpha_{m n} r / R\right), R$ is cylinder radius and $\alpha_{m n}$ the $n$th root of $J_{m}$ ) as unperturbed transverse adiabatic states.

$$
\begin{aligned}
\delta G\left(x_{0}\right)= & -\frac{2 e^{2}}{h}\left(\frac{2 m d^{2}}{\hbar^{2}} S\right)^{2}\left(\frac{d}{R}\right)^{2} \\
& \times\left[\sum_{n m} \frac{1}{\sqrt{\frac{2 m R^{2}}{\hbar^{2}} E_{F}-\alpha_{n m}^{2}}}\left(\frac{J_{m}\left(\alpha_{n m} \frac{x_{0}}{R}\right)}{J_{m+1}\left(\alpha_{n m}\right)}\right)^{2}\right]^{2} .
\end{aligned}
$$

This expression is plotted in Fig. 10 as a function of $x_{0}$ for widths $(=2 R)$ corresponding to steps 1,3 , and 6 .

Now, consider the situation where the scatterer is located close to the side of the neck, see lower panel in Fig. 9. In this case the step structure is maintained somewhat more compared to the situation with the scatterer in the middle of the neck. We see that the steps gets smeared which can be thought of as a decreased effective radius of neck curvature, $R_{\text {eff }}$. The effect reduces quickly as the scatterer is moved towards the exit (entrance) due to the curvature of the neck. This confirms what could be expected, namely, that it is mainly disorder close to the middle of the neck which matters due to the suppression of the wave function near the boundary.
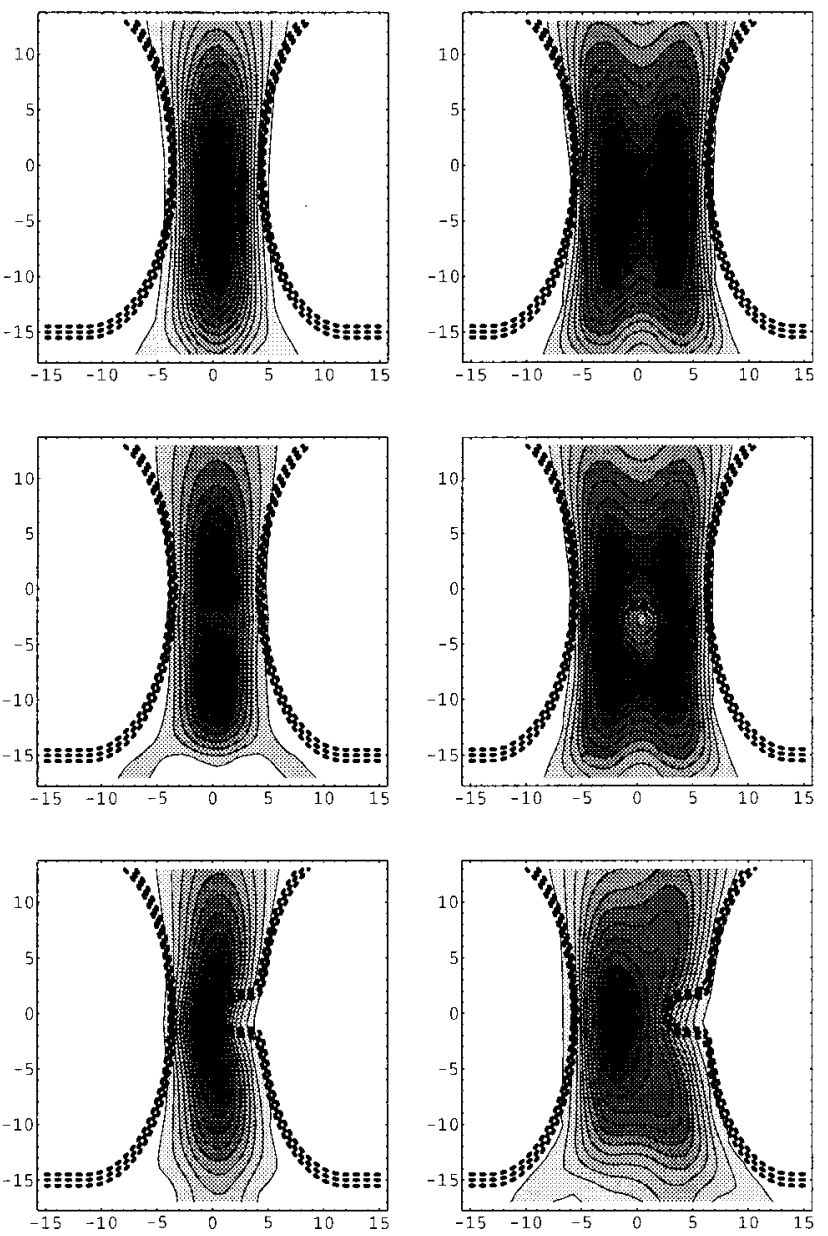

FIG. 11. The contours of the current density in the $z$ direction, $j_{z}$, for widths of 8 (left) and 12 bohr (right). In the upper panel no scatterer is present, in the middle the scatterer is located in the middle, while the scatterer is located on the side in the lower panel. The corresponding conductances are $1.0 G_{0}, 0.3 G_{0}, 0.7 G_{0}$ for the width of $8 \mathrm{bohr}$ and $3.0 G_{0}, 2.7 G_{0}, 2.5 G_{0}$ for the width of $12 \mathrm{bohr}$. The values on the contours are the same except for the middle left where they are scaled by 0.5 .

We have calculated the $j_{z}$ contours and the electron density contours in the $x z$ plane for the incoming scattering states at $E_{F}$, with and without scatterer, for widths of 8 and 12 Bohr corresponding to step 1 and 3 , respectively. These are presented in Figs. 11 and 12. The node structure with one central peak in the case of $G=1 G_{0}$ and two separated peaks for $G=3 G_{0}$ is clearly reflected in both figures. It is seen that the node structure persists in both cases of scatterer position. This must mean that the intrachannel scattering plays a dominant role, since otherwise the structure would be smeared. The interference pattern between incoming and reflected waves in the entrance is seen in the density figure while the diffraction effect is seen in the current density figure in the case where the scatterer is located on the neck side.

In Fig. 13 we present the conductance curves for the same neck just with an elliptical cross section with $\alpha=0.75$ instead of a circular one. This eccentricity splits the degeneracy in angular quantum number $(m= \pm 1)$ of the second mode corresponding to step $3 G_{0}$ and the mode correspond- 

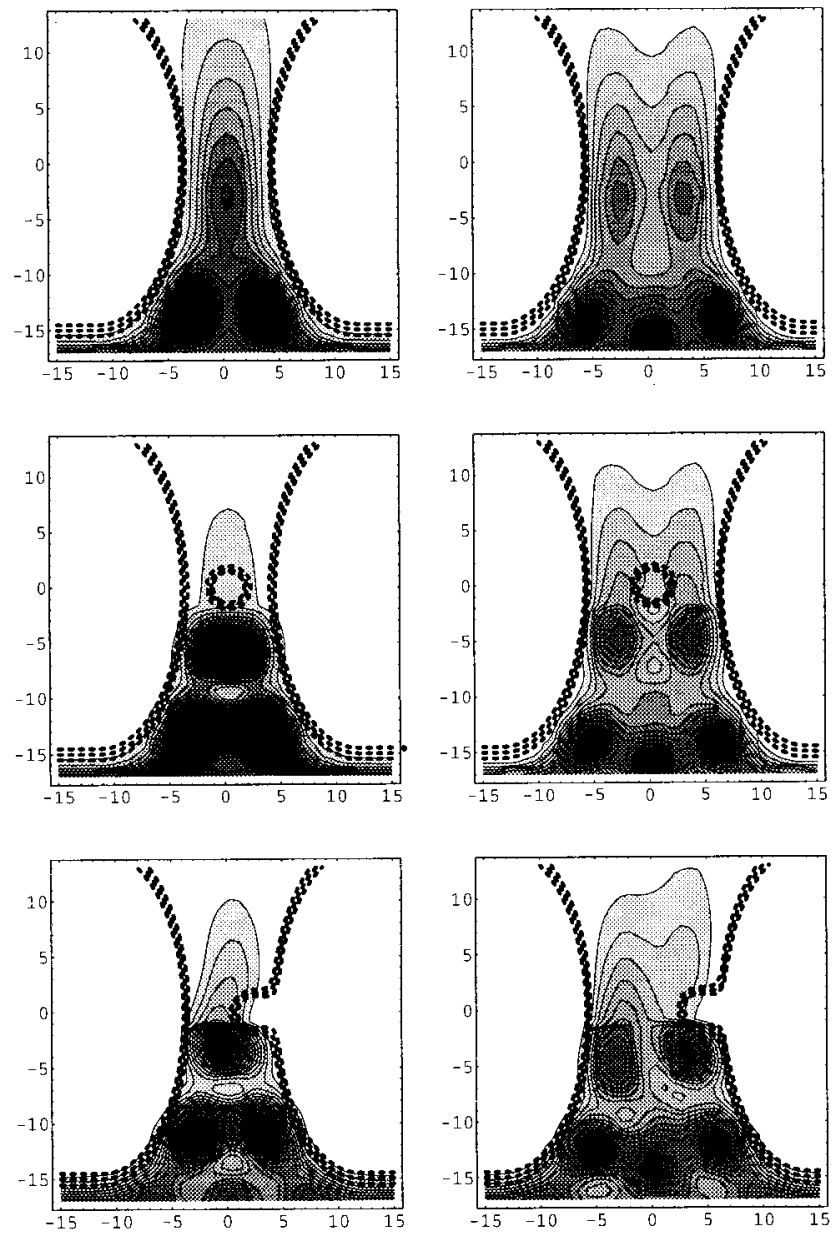

FIG. 12. The contours of the electron density for the incoming scattering state at $E_{F}$ for the same situation as in the previous figure. The node structure inside the neck and the interference pattern between the incoming and reflected waves in the entrance is clearly seen. The persistence of the nodes when the scatterer is introduced reflects that intrachannel scattering must play the dominant role.

ing to step $5 G_{0}(m= \pm 2)$. The disappearance of step $3 G_{0}$ is due to almost degeneracy of two modes. This can be understood if we take the potential to be harmonic. Then the energy level grouping is determined by

$$
\varepsilon_{n_{x}, n_{y}} \propto \alpha n_{x}+n_{y} / \alpha .
$$

For $\alpha=0.75$ this factor yield energies 0., 0.75, 1.33, $1.5,2.08,2.25,2.67$ giving a step sequence for the first steps of 1, 1,2, 2 if we group levels close in energy to account for the step smearing. For $\alpha=1.0$ the corresponding energies are $0 ., 1 ., 1 ., 2 ., 2 ., 2$. , 3. giving a step degeneracy sequence of $1,2,3$. These degeneracies fit well with the findings in Fig. 6 and Fig. 13.

When the scatterer is located in the middle (upper panel) the steps 2 and 4 play the role of step 3 in the case of circular $(g=1)$ cross section and are less affected compared to step 1 . Step 2 is less affected compared to 4 which we can understand if we think of the transverse modes corresponding to $2 G_{0}$ and $4 G_{0}$ having a line of nodes on the short axis

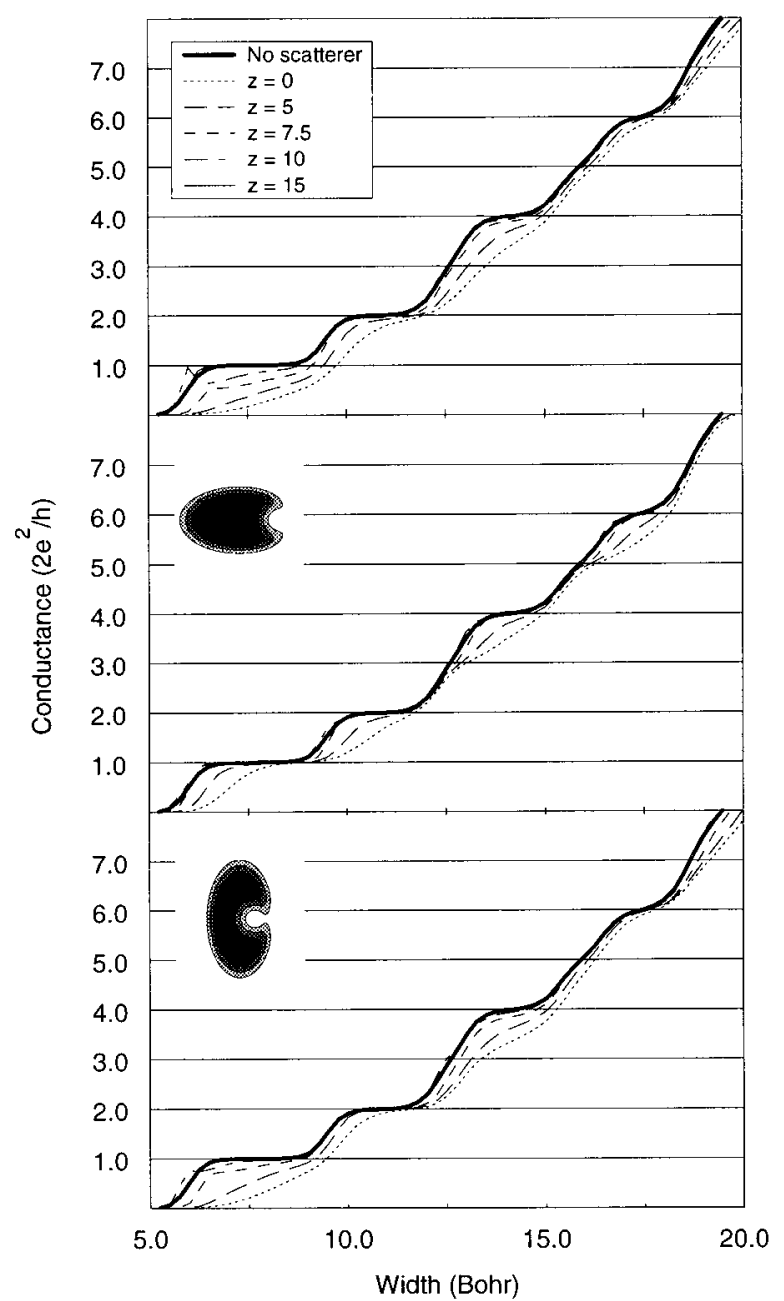

FIG. 13. Same situation as in Fig. 9, but now for a elliptical cross section $(\alpha=0.75)$. Step 3 disappears and steps 2 and 4 appears corresponding to the splitting of the degenerate adiabatic transverse states with $m \pm 1$ and $m \pm 2$. In the upper panel the scatterer is located in the middle while it is on the side in the two lower panels (insets show the neck cross-section contours).

$\left(r_{x}\right)$ and on the long axis $\left(r_{y}\right)$, respectively. Then step 4 will have larger overlap with the scattering potential giving a larger matrix element.

Placing the scatterer on the long $x$ axis (middle panel) we see that step 1 survives but is smeared like in the circular case. Step 2 is destroyed for $z=0$ in this case, but not for the scatterer on the short $y$ axis (lower panel). Here step 1 behaves much like the case where the scatterer is in the middle. Step 4 is strongly affected in both cases, but it is seen that its opening is more delayed when the scatterer is placed on the short $y$ axis (lower panel) compared to the long $x$ axis (middle panel). This we can understand from the nodepicture mentioned before. So in the case of high eccentricity a scatterer located on the side will selectively affect the steps in contrast to the low eccentricity case. We will now turn to the effect of a corrugated neck potential on the transmission.

\section{Corrugated 3D contacts}

The effect of boundary roughness has recently been addressed by Bratkovsky and Rashkeev ${ }^{61}$ for the 3D (circular 


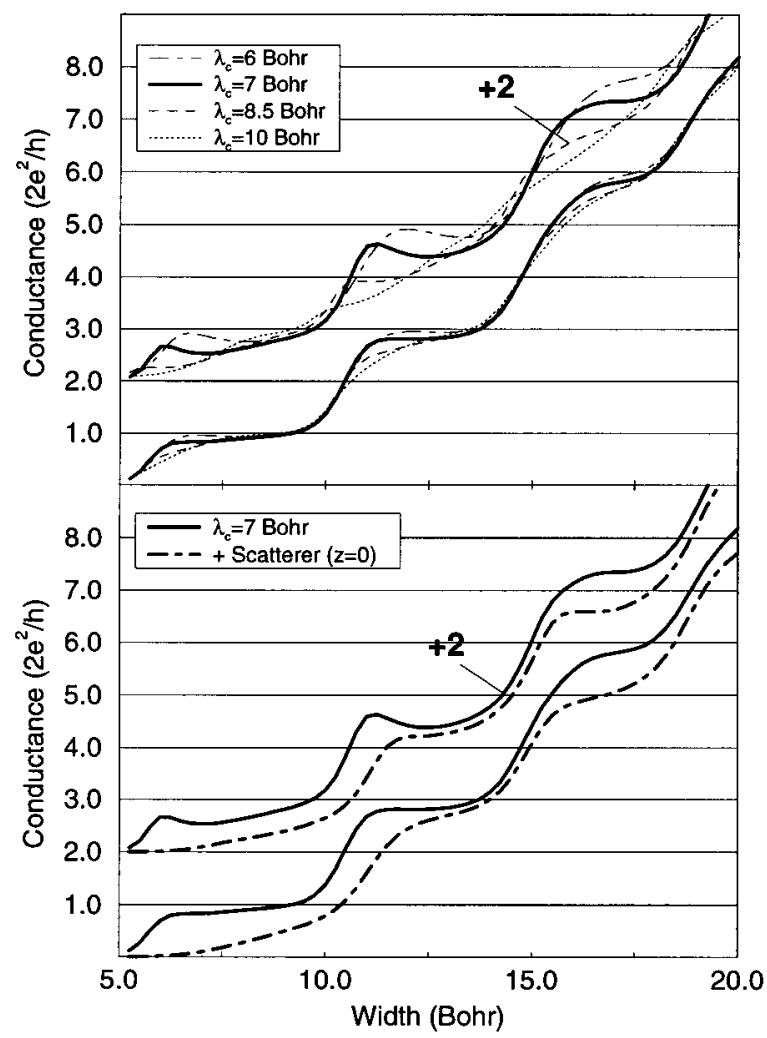

FIG. 14. The conductance curves for corrugated boundary. The upper curves (shifted by $+2 G_{0}$ ) correspond to a corrugation amplitude $A_{c}=2$ bohr, while the lower correspond to $A_{c}=1 \mathrm{bohr}$. The corrugation leads to a downshift of the steps for small $\lambda_{c}$ which decreases with $\lambda_{c}\left(\lambda_{c}=6,7 \mathrm{bohr}\right)$. For larger $\lambda_{c}\left(\lambda_{c}=8.5,10 \mathrm{bohr}\right)$ the steps are smeared corresponding to a smaller $R_{\text {eff }}$ of the neck. Introducing a scatterer in the middle (lower panel) gives a "superposition" of the corrugation effect and the effect of the localized scatterer seen previously.

cross section) case within the free-electron model in a perturbation framework. They include a random noise in the position of the hard-wall neck boundary to simulate the roughness. They conclude that the effect of boundary roughness results in either backscattering destroying conductance quantization or resonant transmission at the opening of new channels. Tekman and $\mathrm{Ciraci}^{57}$ previously have addressed the same problem in the $2 \mathrm{D}$ case. The tight-binding models have been widely used to study boundary as well as internal disorder in $2 \mathrm{D} .{ }^{62,63}$ Here the disorder is modeled by randomizing the on-site energies keeping the nearest neighbor hopping matrix elements constant.

Here we study the effect of boundary corrugation on the conductance curves by modulating the boundary with a term $\left(A_{c} / 2\right) \sin \left(2 \pi z / \lambda_{c}\right)$. In Fig. 14 upper panel we show conductance curves for a corrugated spherically shaped $(R=15$ bohr) neck with a circular cross section. The conductance curves for $\lambda_{c}$ equal to $6,7,8.5$, and $10 \mathrm{bohr}$ and for two different amplitudes $A_{c}=1.0 \mathrm{bohr}$ and $A_{c}=2.0 \mathrm{bohr}$ (shifted by $+2 G_{0}$ ) are shown. In the case of a short corrugation wavelength $\lambda_{c} \leqslant 7.0 \mathrm{bohr}$ (compared to $\lambda_{F}=10 \mathrm{bohr}$ ) a downshift of all the steps is seen, and a downshift decreases with decreasing $\lambda_{c}$. In the case of strong corrugation $\left(A_{c}=2\right.$ bohr) a resonance starts to build up.
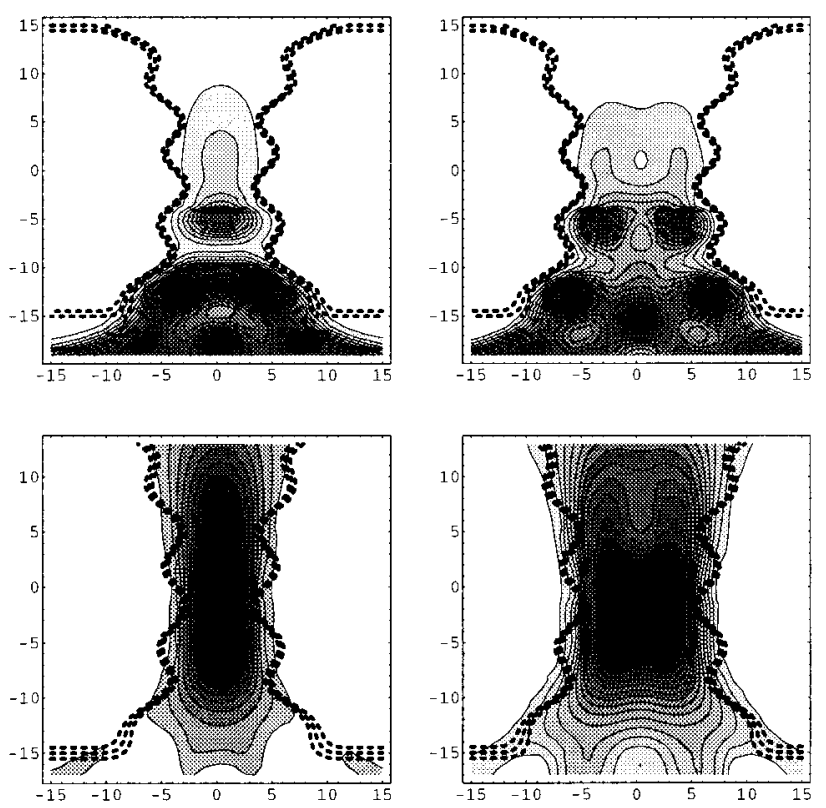

FIG. 15. The electron density (upper) and current density (lower) for the incoming states at $E_{F}$ in the case of a boundary corrugation with amplitude $A_{c}=2 \mathrm{bohr}$ and wavelength $\lambda_{c}=7$ bohr. The conductance is $0.6 G_{0}$ for a width of 8 bohr (left) and $2.45 G_{0}$ for a width of $12 \mathrm{bohr}$ (right) corresponding to a node structure with no node and one node, respectively.

Introducing a scatterer in the middle (lower panel) we see the same effect as in the case of no corrugation just with the new step positions as the starting point. Thus the two scattering mechanisms seem to superimpose. When the corruga-

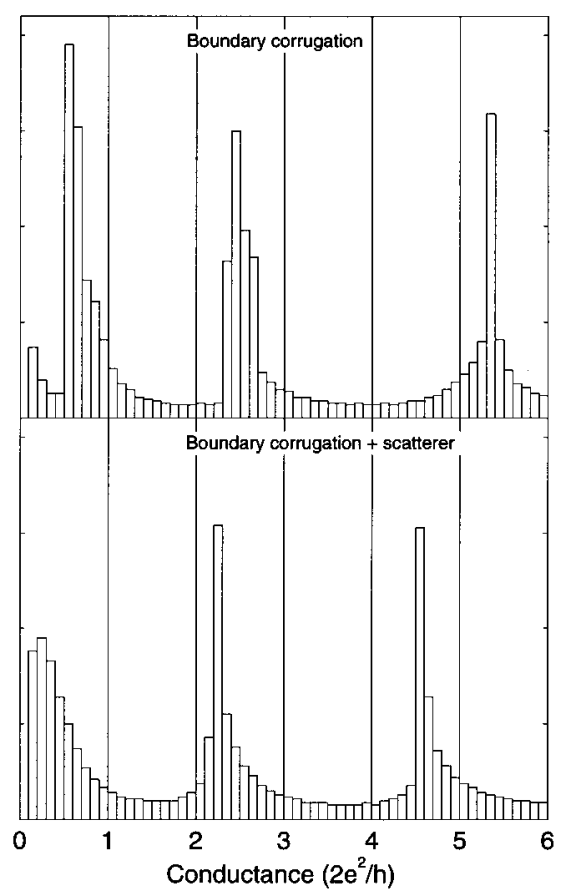

FIG. 16. Upper: The histogram obtained from sampling the conductance during a variation of the width for the strongly corrugated neck $\left(A_{c}=2, \lambda_{c}=7 \mathrm{bohr}\right)$. Lower: The same situation as the upper histogram but now including the scatterer in the middle. 
tion wavelength is increased further $\left(\lambda_{c} \geqslant 7 \mathrm{bohr}\right.$ in upper panel) the steps are smeared corresponding to a smaller $R_{\text {eff }}$. In contrast to the effect of the localized scatterer, which could smear/close single steps selectively, the scattering from boundary corrugation downshift all the steps.

It is remarkable to observe that the step structure remains and that the steps are even sharper in the case with high boundary corrugation and scatterer than in the case with only the scatterer. Thus we can have a step structure shifted away from integer positions in a model where the width is varying continuously, and these steps can still be associated with the quantum modes in the neck structure. In Fig. 15 we show the contour plots of the electron density (upper panel) and $z$ current density (lower panel) for the case of boundary corrugation. We can again conclude that the node structure persists and correlates with the number of conductance channels, even though the conductances are downshifted from the integer positions, see Fig. 15. One can gain further insights ${ }^{64}$ of the nature of the modes and the behavior of the conductance by investigation of the eigenvalves and eigenvectors of $t^{\dagger} t$ and $t t^{\dagger}$.

The quantized conductance is most clearly revealed experimentally by considering conductance histograms from big ensembles of contacts. This is necessary because of the poor reproducibility of the neck structure as discussed earlier. The detailed shapes of the histograms can depend on several factors including overall neck shape, impurity or boundary scattering, and also mechanical instabilities but we shall not go into a discussion of this here. However, we do want to point out that the impurity and boundary scattering discussed above may lead to shifted peaks in a conductance histogram. To illustrate this, we show in the upper panel of Fig. 16 the conductance histogram obtained from a strongly corrugated neck $\left(\lambda_{c}=7.0 \mathrm{bohr}, A_{c}=2 \mathrm{bohr}\right)$. In the lower panel we show the histogram for the scatterer positioned in the middle of this neck. Since we only sample over the neck width, $W$, the histogram is simply inversely proportional to $d G / d W$. The histograms clearly exhibit peaks which are shifted away from the integer positions due to the scattering. However, as the analysis above shows the peaked structure is still due to the well-defined quantum modes perpendicular to the direction of the current.

\section{ACKNOWLEDGMENTS}

Stimulating discussions with Flemming Besenbacher, Kim Hansen, Erik Lægsgaard, Horia Metiu, Lars Olesen, Ivan Stensgaard, and Mads R. Sørensen are gratefully acknowledged. Martijn Krans is acknowledged for kindly sending us Fig. 3 taken from his thesis.(Ref. 20). The Center for Atomic-scale Materials Physics is sponsored by the Danish National Research Foundation. Further funding is obtained from the Danish Research Councils through the Center for Nano-Tribology and from National Science Foundation Grant Nos. DMR-9419506 and 9501775.
${ }^{1}$ D. A. Wharam, T. J. Thornton, R. Newbury, M. Pepper, H. Ahmed, J. E. F. Frost, D. G. Hasko, D. C. Peacock, D. A. Ritchie, and G. A. C. Jones, J. Phys. C 21, L209 (1988).

${ }^{2}$ B. J. van Wees, H. van Houten, C. W. J. Beenakker, J. G. Williamson, L. P. Kouwenhoven, D. van der Marel, and C. T. Foxon, Phys. Rev. Lett. 60, 848 (1988).

${ }^{3}$ J. K. Gimzewski and R. Möller, Phys. Rev. B 36, 1284 (1987).

${ }^{4}$ U. Dürig, O. Züger, and D. W. Pohl, Phys. Rev. Lett. 65, 349 (1990).

${ }^{5}$ L. Kuipers and J. W. M. Frenken, Phys. Rev. Lett. 70, 3907 (1993).

${ }^{6}$ J. I. Pascual, J. Méndez, J. Gómez-Herrero, A. M. Baró, N. García, and Vu Thien Binh, Phys. Rev. Lett. 71, 1852 (1993).

${ }^{7}$ N. Agrait, J. G. Rodrigo, and S. Vieira, Phys. Rev. B 47, 12345 (1993).

${ }^{8}$ L. Olesen, E. Lægsgaard, I. Stensgaard, F. Besenbacher, J. Schiøtz, P. Stoltze, K. W. Jacobsen, and J. K. Nørskov, Phys. Rev. Lett. 72, 2251 (1994).

${ }^{9}$ V. V. Dremov, S. Yu. Shapoval, and E. V. Sukhorukov, Phys. Low-Dim. Struct. 11/12, 29 (1994).

${ }^{10}$ M. Brandbyge, J. Schiøtz, M. R. Sørensen, P. Stoltze, K. W. Jacobsen, J. K. Nørskov, L. Olesen, E. Lægsgaard, I. Stensgaard, and F. Besenbacher, Phys. Rev. B 52, 8499 (1995).

${ }^{11}$ J. I. Pascual, J. M. Méndez, J. Gómez-Herrero, A. M. Baró, N. García, U. Landman, W. D. Luedtke, E. N. Bogachek, and H. P. Cheng, Science, 267, 24 (1995).

${ }^{12}$ N. Agrait G. Rubio, and S. Vieira, Phys. Rev. Lett. 74, 3995 (1995).

${ }^{13}$ G. Rubio, N. Agrait and S. Vieira, Phys. Rev. Lett. 76, 2302 (1996).
${ }^{14}$ C. Sirvent, J. G. Rodrigo, N. Agrait and S. Vieira, Physica B 218, 238 (1996).

${ }^{15}$ Z. Gai, Y. He, H. Yu, and W. S. Yang, Phys. Rev. B 53, 1042 (1996).

${ }^{16}$ J. M. Krans, C. J. Muller, I. K. Yanson, Th. C. M. Govaert, R. Hesper, and J. M. van Ruitenbeek, Phys. Rev. B 48, 14721 (1993).

${ }^{17}$ J. M. Krans and J. M. van Ruitenbeek, Phys. Rev. B 50, 17659 (1994).

${ }^{18}$ J. M. Krans, J. M. van Ruitenbeek, V. V. Fisun, I. K. Yanson, and L. J. de Jongh, Nature (London) 375, 767 (1995).

${ }^{19}$ C. J. Muller, J. M. Krans, T. N. Todorov, and M. A. Reed, Phys. Rev. B 53, 1022 (1996).

${ }^{20}$ J. M. Krans, Ph.D. Thesis, Leiden, 1996.

${ }^{21}$ J. M. Krans, J. M. van Ruitenbeek, and L. J. de Jongh, Physica B 218, 228 (1996).

${ }^{22}$ D. P. E. Smith, Science 269, 21 (1995).

${ }^{23}$ L. Olesen, M. Brandbyge, M. R. Sørensen, K. W. Jacobsen, E. Lægsgaard, I. Stensgaard, and F. Besenbacher, Phys. Rev. Lett. 76, 1485 (1996).

${ }^{24}$ J. B. Pethica and A. P. Sutton, J. Vac. Sci. Technol. A 6, 2490 (1988).

${ }^{25}$ M. R. Sørensen, H. Jónson, and K. W. Jacobsen, Phys. Rev. Lett. (to be published).

${ }^{26}$ J. L. Costa-Krämer, N. García, P. García-Mochales, and P. A. Serena, Surf. Sci. Lett. 342, L1144 (1995); 349, (E)L138 (1996).

${ }^{27}$ U. Landman, W. D. Luedtke, B. E. Salisbury, and R. L. Whetten, Phys. Rev. Lett. 77, 1362 (1996)

${ }^{28}$ K. Hansen, E. Lægsgaard, I. Stensgaard, and F. Besenbacher (unpublished). 
${ }^{29}$ J. M. Krans, C. J. Muller, N. van der Post, F. R. Postma, A. P. Sutton, T. N. Todorov, and J. M. van Ruitenbeek, Phys. Rev. Lett. 74, 2146 (1995).

${ }^{30}$ L. Olesen, E. Lægsgaard, I. Stensgaard, F. Besenbacher, J. Schiøtz, P. Stoltze, K. W. Jacobsen, and J. K. Nørskov, Phys. Rev. Lett. 74, 2147 (1995).

${ }^{31}$ Walt A. de Heer, Rev. Mod. Phys. 65, 611 (1993).

${ }^{32}$ U. Landman, W. D. Luedtke, N. A. Burnham, and R. J. Colton, Science 248, 454 (1990).

${ }^{33}$ A. P. Sutton, J. B. Pethica, H. Rafii-Tabar, and J. A. Nieminen, in Electron Theory in Alloy Design, edited by D. G. Pettifor and A. H. Cottrell (Institute of Materials, London, 1992), Chap 7.

${ }^{34}$ T. N. Todorov and A. P. Sutton, Phys. Rev. Lett. 70, 2138 (1993).

${ }^{35}$ A. M. Bratkovsky, A. P. Sutton, and T. N. Todorov, Phys. Rev. B 52, 5036 (1995).

${ }^{36}$ R. Landauer, IBM J. Res. Dev. 1, 223 (1957); Phys. Scr., T42, 110 (1992); Y. Imry, in Directions in Condensed Matter Physics edited by by G. Grinstein and G. Mazendko (World Scientific, Singapore, 1986), p. 101; C. W. J. Beenakker and H. van Houten, in Solid State Physics: Advances in Research and Applications, edited by H. Ehrenreich and D. Turnbull (Academic, New York, 1991), Vol. 44.

${ }^{37}$ R. Landauer, J. Phys. C 1, 8099 (1989).

${ }^{38}$ M. Büttiker, in Electronic Properties of Multilayers and LowDimensional Semiconductor Structures, edited by J. M. Chamberlain et al. (Plenum Press, New York, 1990).

${ }^{39}$ L. I. Glazman, G. B. Lesovik, D. E. Khmel'nitskii, and R. I. Shekhter, Pis'ma Zh. Éksp. Teor. Fiz. 48, 218 (1988) [JETP Lett. 48, 238 1988.]

${ }^{40}$ A. Yacoby and Y. Imry, Phys. Rev. B 41, 5341 (1990).

${ }^{41}$ F. A. Maaø I. V. Zozulenko, and E. H. Hauge, Phys. Rev. B 50, 17320 (1994).

${ }^{42}$ E. N. Bogachek, A. N. Zagoskin, and I. O. Kulik, Sov. J. Low Temp. Phys. 16, 796 (1990).
${ }^{43}$ M. Yosefin and M. Kaveh, Phys. Rev. Lett. 64, 2819 (1990).

${ }^{44}$ J. A. Torres, J. I. Pascual, and J. J. Sáenz, Phys. Rev. B 49, 16581 (1994).

${ }^{45}$ Y. V. Sharvin, Zh. Éksp. Teor. Fiz. 48, 984 (1965) [Sov. Phys. JETP 21, 655 (1965)].

${ }^{46}$ A. G. Scherbakov, E. N. Bogachek, and U. Landman, Phys. Rev. B 53, 4054 (1996).

${ }^{47}$ J. A. Torres and J. J. Sáenz, Physica B 218, 234 (1996).

${ }^{48}$ J. A. Torres and J. J. Sáenz, Phys. Rev. Lett. 77, 224 (1996).

${ }^{49}$ H. Kasai, T. Kakuda, and A. Okiji, Surf. Sci. 363, 428 (1996).

${ }^{50}$ T. N. Todorov, G. A. D. Briggs, and A. P. Sutton, J. Phys. C 5, 2389 (1993).

${ }^{51}$ D. M. Newns, Phys. Rev. 178, 1123 (1969).

${ }^{52}$ K. Hirose and M. Tsukada, Phys. Rev. B 51, 5278 (1995); Phys. Rev. Lett. 73, 150 (1994); J. Vac. Sci. Technol. B 12, 2164 (1994).

${ }^{53} \mathrm{Ph}$. Lambin and J. P. Vigneron, J. Phys. A 13, 1815 (1981), J. P. Vigneron and $\mathrm{Ph}$. Lambin, ibid. 13, 1135 (1980).

${ }^{54}$ H. Q. Nguyen, P. H. Cutler, T. E. Feuchtwang, N. Miskovsky, and A. A. Lucas, Surf. Sci. 160, 331 (1985).

${ }^{55}$ E. G. Haanappel and D. van der Marel, Phys. Rev. B 39, 5484 (1989); D. van der Marel and E. G. Haanappel, ibid. 39, 7811 (1989).

${ }^{56}$ J. B. Pendry, A. Prêtre, P. J. Rous, and L. Martìn-Moreno, Surf. Sci. 244, 160 (1991).

${ }^{57}$ E. Tekman and S. Ciraci, Phys. Rev. B 43, 7145 (1991).

${ }^{58}$ P. F. Bagwell, Phys. Rev. B 41, 10354 (1990).

${ }^{59}$ N. D. Lang, Phys. Rev. B 52, 5335 (1995).

${ }^{60}$ L. I. Glazman and M. Jonson, Phys. Rev. B 44, 3810 (1991).

${ }^{61}$ A. M. Bratkovsky and S. N. Rashkeev, Phys. Rev. B 53, 13074 (1996).

${ }^{62}$ K. Nikolić and A. MacKinnon, Phys. Rev. B 50, 11008 (1994).

${ }^{63}$ P. García-Mochales, P. A. Serena, N. García, and J. L. CostaKrämer, Phys. Rev. B 53, 10268 (1996).

${ }^{64}$ M. Brandbyge and K. W. Jacobsen (unpublished). 